\title{
Consenso sobre el manejo de eventos de aparente amenaza a la vida del lactante (ALTE)
}

\author{
Comisión de Sueño, Sociedad Chilena \\ de Neumología Pediátrica 2013
}

\author{
Miembros: PABLO E. BROCKMANN V. ${ }^{1}$ (Preside comisión), SELIM ABARA E. ${ }^{2}$, CAROLINA CAMPOS O. ${ }^{3}$, \\ NILS L. HOLMGREN P. ${ }^{2}$, SOLEDAD MONTES F. ${ }^{4}$, HERNÁN SEPÚLVEDA R. ${ }^{5}$, DANIEL ZENTENO A. ${ }^{6}$ \\ 1. Escuela de Medicina, Facultad de Medicina, Pontificia Universidad Catolica de Chile. \\ 2. Clínica Alemana de Santiago, Universidad del Desarrollo. \\ 3. Hospital Dr. Sótero del Río. \\ 4. Hospital San Borja Arriarán. \\ 5. Hospital Luis Calvo Mackenna, Universidad de Chile. \\ 6. Hospital Guillermo Grant Benavente, Universidad de Concepción.
}

\begin{abstract}
Consensus on evaluation and management of apparent life-threatening events in infancy (ALTE)

Apparent life threatening events are defined as an acute episode in which the observer fears an infant $<1$ year may die. ALTE is characterized by some combination of apnea, color or muscle tone change, chocking and has to be followed by cardiorespiratory reanimation. The present consensus paper reviews international and national evidence concerning diagnosis, etiologies, hospitalization criteria and indications for home monitoring. (Key words: Apparent life threatening event, ALTE, sudden death, apnea).

Rev Chil Pediatr 2014; 85 (3): 378-387
\end{abstract}

\section{RESUMEN}

Un Evento de Aparente Amenaza de la Vida o ALTE (del inglés: Apparent Life Threatening Event) corresponde a un episodio agudo que ante los ojos del observador pone en riesgo la vida de un lactante menor de 1 año. Debe presentar la combinación de 2 o más de los criterios siguientes: apnea / cambio de color / alteración del tono / atoro e implicar la necesidad de algún tipo de maniobras para reanimar. En el presente consenso sobre el manejo de un ALTE se revisaron la evidencia internacional y nacional respecto al enfoque diagnóstico, estudio etiológico, criterios y duración de hospitalización y las indicaciones de monitorización domiciliaria.

(Palabras clave: Evento de Aparente Amenaza de la Vida, ALTE, apnea, muerte súbita).

Rev Chil Pediatr 2014; 85 (3): 378-387

Recibido el 20 de enero de 2014, aceptado para publicación el 28 de enero de 2014.

Este trabajo cumple con los requisitos sobre consentimiento /asentimiento informado, comité de ética, financiamiento, estudios animales y sobre la ausencia de conflictos de intereses según corresponda.

Correspondencia a:

Dr. Pablo E. Brockmann

pbrockmann@med.puc.cl 


\section{Introducción}

Un Evento de Aparente Amenaza de la Vida o ALTE (del inglés: Apparent Life Threatening Event) en un lactante pequeño, actualmente continúa siendo un desafío para el médico que lo enfrenta. A pesar del gran avance en recursos diagnósticos y de seguimientos en las últimas décadas, que nos han permitido tener mucha información y entender los eventos que pueden producir un ALTE; enfrentados al caso individual, el médico debe tomar difíciles e importantes decisiones, a veces con muchas interrogantes ${ }^{1}$.

A mediados de los años sesenta, se inició el uso de los monitores de apnea para el manejo de esta patología en prematuro, extendiéndose posteriormente la monitorización cardiorrespiratoria para el manejo de diversas condiciones agudas y crónicas. A fines de los setenta, se iniciaron las primeras investigaciones sobre las posibles etiologías del Síndrome de Muerte Súbita del Lactante (SMSL) o SIDS (del inglés: Sudden Infant Death Syndrome), con la hipótesis de que estos niños, podrían haber presentado un episodio previo al evento final, con las características de lo que hoy conocemos como ALTE ${ }^{1}$.

Las investigaciones continuaron su desarrollo en los ochenta y mediados de los noventa sobre aspectos fisiológicos, variables bioquímicas y metabólicas en recién nacidos con historia de ALTE, de manera de aclarar las causas y dilucidar su relación con el SIDS. La posible relación entre ALTE y SMSL nunca ha sido demostrada, existiendo evidencias que ambas condiciones podrían no estar relacionadas ${ }^{1}$. En el año 1986, el National Institute of Child Health and Human Development acuña el término ALTE a un episodio que atemoriza al observador, caracterizado por una combinación de apnea central y ocasionalmente obstructiva, cambios en la coloración (habitualmente cianosis, palidez o rubicundez), asociado a alteraciones del tono muscular (hipotonía), con ahogo o sofocación ${ }^{2}$.

Esta definición, con un componente subjetivo importante, describe una multiplicidad de factores observados, producto de numerosas etiologías, algunos que deben ser sos- pechados desde el inicio, como infecciones bacterianas graves, convulsiones, abuso infantil, trastornos metabólicos y apneas graves con hipoxemia. En un porcentaje que varía de un $20 \%$ a casi la mitad de los casos, no se encuentra etiología, lo que a veces es referido como "ALTE idiopático"3. Este fenómeno queda de manifiesto en una revisión sistemática publicada el 2004, en la cual se analizaron un total de 2.912 citas bibliográficas, de las cuales se incluyeron 8 estudios $(n=643$ lactantes). En todos los estudios incluidos, se hospitalizaron a los pacientes y se obtuvieron más de 728 diagnósticos distintos al momento del alta ${ }^{4}$.

Hacia el año 2000 se habían estudiado diversas condiciones que podían conducir a un ALTE, pero en los últimos años, las investigaciones han disminuido posiblemente por la subjetividad de las observaciones, el uso de terminología distinta para describir los mismos episodios y la multiplicidad de causas.

Se ha estimado que el $7 \%$ de los casos de SMSL son precedidos por un ALTE $^{5}$. La incidencia de ALTE es variable; calculado entre 0,46 y 10 por cada 1.000 nacidos vivos, representa el $0,8 \%$ y el $1 \%$ de todas las consultas en Servicios de Urgencias de menores de 1 año y hasta un $2 \%$ del total de hospitalizaciones pediátricas ${ }^{6}$. Los eventos de ALTE con resultado de muerte son globalmente un 7,6\% En nuestro país, se cuenta con los datos de Santiago y Concepción respecto a prevalencia y etiología de ALTE $^{8,9}$, la cual fue similar a los datos publicados en el extranjero.

Es importante señalar que el clínico puede enfrentarse a un niño en buenas condiciones al momento de consultar (posterior al evento) en el $80 \%$ de los casos, por lo que no es fácil decidir cuándo hospitalizar y estudiar ${ }^{10}$, debiendo realizar una detallada historia clínica, identificar y tratar cualquier trastorno subyacente, educar y apoyar a la familia, y organizar el estudio y seguimiento $^{3}$. Con frecuencia se realizan estudios de alto costo, que rara vez contribuyen a determinar la causa del ALTE. Sólo un 7\% a 16\% de los niños ingresados por ALTE necesitan una intervención significativa durante la hospitalización ${ }^{6}$. 


\section{Objetivos}

El objetivo del presente consenso fue revisar la mejor evidencia tanto internacional como nacional referente al tema ALTE. Mediante la revisión de forma independiente de los estudios relevantes al respecto, se llegó a un consenso de recomendaciones de expertos en el tema, sugiriéndose una pauta de estudio, manejo y tratamiento que permita tratar de la mejor manera posible este difícil cuadro clínico.

\section{Definición}

La definición de ALTE se ha tomado del acuerdo publicado por el National Institute of Child Health and Human Development en $1986^{2}$. Las publicaciones internacionales y nacionales han citado y seguido esta definición desde esa fecha, por lo que planteamos que ésta debiera de ser la definición diagnostica única que se utilice para el manejo de los pacientes con ALTE. ALTE debería ser un diagnóstico operativo de ingreso, pero no debería ser considerado como un diagnóstico de egreso si se ha logrado identificar la causa.

Como se mencionó en la introducción, ALTE corresponde a un evento súbito con las siguientes características:

1. Su definición es subjetiva y depende de la impresión del cuidador, frecuentemente madre o padre.

2. Es un diagnóstico operativo exclusivo de lactantes menores de 1 año.

3. Corresponde a un evento agudo que ante los ojos del observador pone en riesgo la vida del niño.

4. Debe presentar la combinación de más de 1 de los criterios siguientes: Apnea / cambio de color / alteración del tono / atoro.

5. Implica la necesidad de algún tipo de maniobras para reanimar. Este último punto es difícil, ya que los cuidadores habitualmente no están capacitados o entrenados en maniobras de reanimación cardiopulmonar formales, por lo que la estimulación que se realizó al lactante (agitar, soplar, remecer, entre otros) merece especial atención al momento de realizar la anamnesis.
Se sugiere expresamente no utilizar los términos "muerte súbita frustra", "apnea", "crisis de apnea" o similares que asimilen el ALTE con otras patologías, que no tienen necesariamente asociación fisiopatológica.

\section{Factores de riesgo}

Factores de riesgo para presentar un ALTE $^{7}$ :

1. Tabaquismo materno.

2. Prematurez.

Factores de riesgo de potencial gravedad de un ALTE (ALTE severo, recurrente y/o asociado a una enfermedad subyacente $)^{10}$ :

1. Prematurez.

2. Edad gestacional corregida $<43$ semanas, aumenta hasta en 5,2 veces el riesgo de repetir un evento durante la hospitalización ${ }^{10,11}$. Múltiples ALTEs (en cluster y/o recurrente después del alta), muchos estudios reportan mayor riesgo de una enfermedad de base, ej. maltrato infantil y convulsiones.

3. Síntomas de infección respiratoria, un estudio mostró que tienen mayor probabilidad de repetir eventos que inicialmente pueden ser interpretados como ALTE ${ }^{10-11}$.

4. Requerimiento de reanimación cardiopulmonar vigorosa o formal para abortar el episodio ${ }^{10}$.

5. Hermanos o familiares directos con antecedentes de muerte súbita ${ }^{12}$.

6. Antecedentes sociales de riesgo ${ }^{12}$.

La edad como factor de riesgo principal para un ALTE ha sido investigada en varios estudios, en una revisión sistemática de 2013 por Tieder y cols. ${ }^{10}$, señalando que a menor edad aumenta hasta en 5,2 veces el riesgo de repetir ALTE durante la hospitalización, lo cual es más significativo en menores de 6 meses. Esto está en línea con los resultados de los dos estudios chilenos de ALTE publicados hasta la fecha, en los cuales se pudo demostrar que la mayor parte de los casos sucedía antes de los 6 meses de vida, con un alza de los casos alrededor del $2^{\circ}$ mes de vida ${ }^{8,9}$. 


\section{Etiologías}

Se han descrito múltiples causas de ALTE, asociando estos eventos a infecciones respiratorias virales, reflujo gastroesofágico (RGE), convulsiones, alteraciones anatómicas de la vía aérea, causas cardiológicas, metabólicas o idiopáticas ${ }^{10}$. El diagnóstico diferencial de ALTE publicado en la literatura internacional, varía según el estudio realizado y los medios disponibles en cada uno de los centros en los cuales se efectuó.

Tieder y cols. ${ }^{10}$, describen las etiologías más frecuentes de ALTE, dentro de las cuales se encuentra RGE o regurgitación, infecciones virales y convulsiones (tabla 1). Es fundamental la sospecha de maltrato o Síndrome de Münchhausen por poder, ante ALTEs múltiples (cluster o recurrente post-alta), llegando a ser responsable de $0,4-11 \%$ de los ALTE $^{10}$. Cabe recordar que la primera demostración de apneas asociadas a ALTE recurrente fue publicada en 1971 por Steinschneider en los Estados Unidos ${ }^{13}$, resultando años después en una confesión de infanticidio múltiple por la madre de los menores. Los hallazgos del examen físico y de una historia sugerente de un ALTE no orgánico asociado a maltrato infantil son: múltiples ALTE evidenciado por 1 solo testigo, historia de falta de respuesta a la reanimación, hipotonía, muerte súbita de hermano, equimosis facial o hemorragia $u$ otros signos de maltrato al examen físico.

Tabla 1. síntomas, hallazgos y estudio sugerido

\begin{tabular}{|c|c|c|}
\hline Causas & Síntomas y hallazgos & Estudio sugerido \\
\hline $\begin{array}{l}\text { Gastrointestinales } \\
\text { RGE } \\
\text { Aspiración }\end{array}$ & $\begin{array}{l}\text { - Vómitos } \\
\text { - Atoros o tos con alimentación }\end{array}$ & $\begin{array}{l}\text { - Phmetría } \\
\text { - Estudio video-deglución } \\
\text { Evaluación fonoaudiológica }\end{array}$ \\
\hline $\begin{array}{l}\text { Respiratorias } \\
\text { Infección viral/Pertussis } \\
\text { Aspiración /cuerpo extraño } \\
\text { Alteración anatómica vía aérea }\end{array}$ & $\begin{array}{l}\text { - Coriza, tos, sibilancias, fiebre, hipotermia } \\
\text { - Historia ingestión cuerpo extraño } \\
\text { - Estridor, dificultad con alimentación, } \\
\text { dismorfias, especialmente cráneo-faciales }\end{array}$ & $\begin{array}{l}\text { - Panel viral, IFD viral } \\
\text { PCR Bordetella } \\
\text { - Evaluación endoscópica vía aérea } \\
\text { - Evaluación endoscópica vía aérea }\end{array}$ \\
\hline $\begin{array}{l}\text { Trauma } \\
\text { Munchhausen por poder }\end{array}$ & $\begin{array}{l}\text { - Historia de trauma, sangre en boca o nariz } \\
\text { - ALTE previo, hermano con SIDS, historia } \\
\text { discordante }\end{array}$ & $\begin{array}{l}\text { - Evaluación clínica radiológica } \\
\text { - Descartar maltrato } \\
\text { Sospecha clínica }\end{array}$ \\
\hline Neurológicas/convulsivas & $\begin{array}{l}\text { - Pérdida de conciencia } \\
\text { Desviación de la mirada } \\
\text { Convulsión, Hipotonía/hipertonía } \\
\text { Microcefalia/macrocefalia } \\
\text { Dismorfias }\end{array}$ & $\begin{array}{l}\text { - EEG } \\
\text { Eco cerebral } \\
\text { TAC / RNM de cerebro } \\
\text { Glicemia; Ca, P, Mg, ELP } \\
\text { Estudio Metabólico }\end{array}$ \\
\hline Apnea emotiva & $\begin{array}{l}\text { - Clínica sugerente sin otros hallazgos } \\
\text { (en vigilia) }\end{array}$ & - Descartar anemia asociada \\
\hline Enfermedades metabólicas & $\begin{array}{l}\text { - Historia familiar } \\
\text { Convulsiones } \\
\text { Problemas con alimentación } \\
\text { Compromiso de conciencia } \\
\text { Dismorfias }\end{array}$ & $\begin{array}{l}\text { - Glicemia, } \\
\text { Lactato, amonio, piruvato } \\
\text { Aminoacidemia, aminoaciduria, etc. }\end{array}$ \\
\hline $\begin{array}{l}\text { Cardiovascular: } \\
\text { Cardiopatía congénita/arritmias }\end{array}$ & $\begin{array}{l}\text { - Alimentación con dificultad } \\
\text { Diaforesis } \\
\text { Cianosis central, Síncope }\end{array}$ & $\begin{array}{l}\text { - Electrocardiograma( QTc) } \\
\text { Holter } 24 \text { horas } \\
\text { Ecocardiograma Doppler }\end{array}$ \\
\hline $\begin{array}{l}\text { Infecciosas: } \\
\text { Meningitis / Septicemia / ITU }\end{array}$ & - Fiebre, Hipotermia, Letargia y/o Shock & $\begin{array}{l}\text { - Hemograma, PCR } \\
\text { Hemocultivos, Orina completa, urocultivo } \\
\text { Punción lumbar }\end{array}$ \\
\hline Medicamentos o tóxicos & $\begin{array}{l}\text { - Compromiso de conciencia } \\
\text { Letargia, antecedentes previos }\end{array}$ & - Screening toxicológico en sangre y/o orina \\
\hline
\end{tabular}


La tabla 1 resume los síntomas y hallazgos sugerentes de las causas más frecuentes de ALTE. No obstante a lo anterior, entre un 20 a $30 \%$ de los casos no se logra llegar a una etiología clara pese a un estudio detallado.

\section{Criterios de hospitalización}

La gran variabilidad etiológica y de presentación de los ALTE hace difícil establecer consideraciones estándar para determinar hospitalización, y pese a la existencia de recomendaciones, la gran mayoría de estos pacientes se hospitaliza. La decisión de hospitalización es responsabilidad del médico que recibe al paciente, quien tomando en consideración la presentación clínica y repercusión que generó el evento en los padres, define el tratamiento ${ }^{9,14}$.

Se estima que el $12-14 \%$, presenta recurrencia, la cual se da en $85 \%$ durante las primeras 24 h después del episodio inicial ${ }^{11,15}$.

La necesidad de Reanimación Cardiopulmonar (RCP) o estimulación vigorosa, es considerada por algunos autores como indicación absoluta de hospitalización; pese a la subjetividad y/o incerteza de la real necesidad de ésta $^{12,16}$.

Los factores de riesgo deben ser considerados al momento de decidir el alta a domicilio desde el servicio de urgencia; además de condiciones patológicas asociadas a mayor riesgo de ALTE y/o recurrencia (por ej.: malformaciones craneo-faciales, genopatías, enfermedades neuromusculares y cardiovasculares $)^{6}$.

\section{Criterios de hospitalización}

- Empleo de RCP o estimulación vigorosa.

- Antecedentes de prematurez. Menos de 43 semanas de edad gestacional corregida.

- Menor de 2 meses.

- Recurrencia.

- Hermano fallecido por SMSL.

- Condición patológica asociada.

- Sospecha de maltrato infantil.

- Factores de riesgo social.

\section{Lugar de hospitalización}

La hospitalización debe ser en una unidad donde se puede realizar una monitorización cardiorespiratoria continua y eventual reani- mación adecuada y segura, conforme a la realidad de los distintos establecimientos de salud.

El paciente que requirió RCP evidentemente necesaria y/o que presenta nuevos eventos constatados por personal de salud, debe ser siempre manejado en unidad de cuidados intensivos pediátricos.

\section{Objetivos de Hospitalización $n^{12,17}$}

- Monitorización, observación clínica y tratamiento de recurrencias.

- Realizar de estudios etiológicos generales y específicos.

- Determinación de tratamientos específicos.

- Educación a padres en reanimación cardiopulmonar y medidas que disminuyen SMSL.

\section{Criterios de alta hospitalaria}

- Monitorización mínima de 24 h sin nuevos eventos, con resultados de estudios dentro de rango normal.

- ALTE secundario: Una vez tratada patología que causó el ALTE.

- ALTE idiopático: por lo menos con estudios iniciales conocidos, dentro de rango normal.

- Padres educados en como enfrentar a nuevo episodio de ALTE y entrenados en RCP.

- Identificación y corrección de factores de riesgo de maltrato infantil.

- Educación de Medidas para un dormir seguro (Factores riesgo SMSL, se recomienda utilización de "tarjetas de cuna" [figura 1]).

\section{Objetivos del control ambulatorio ${ }^{12}$}

- Confirmar la etiología atribuida a los eventos.

- Descartar comorbilidad asociada.

- Derivar a subespecialidades si procede.

- Mitigar el estrés de familiares frente a dudas que surgen y/o no resueltas durante hospitalización.

- Reforzar educación sobre factores de riesgo de SMSL.

- Control de monitorización domiciliaria.

- Descartar evidencias de maltrato infantil.

\section{Asociación con muerte súbita}

No existe evidencia que sustente que el ALTE se asocie, ni sea precursor de $\mathrm{SMSL}^{7,18}$. 


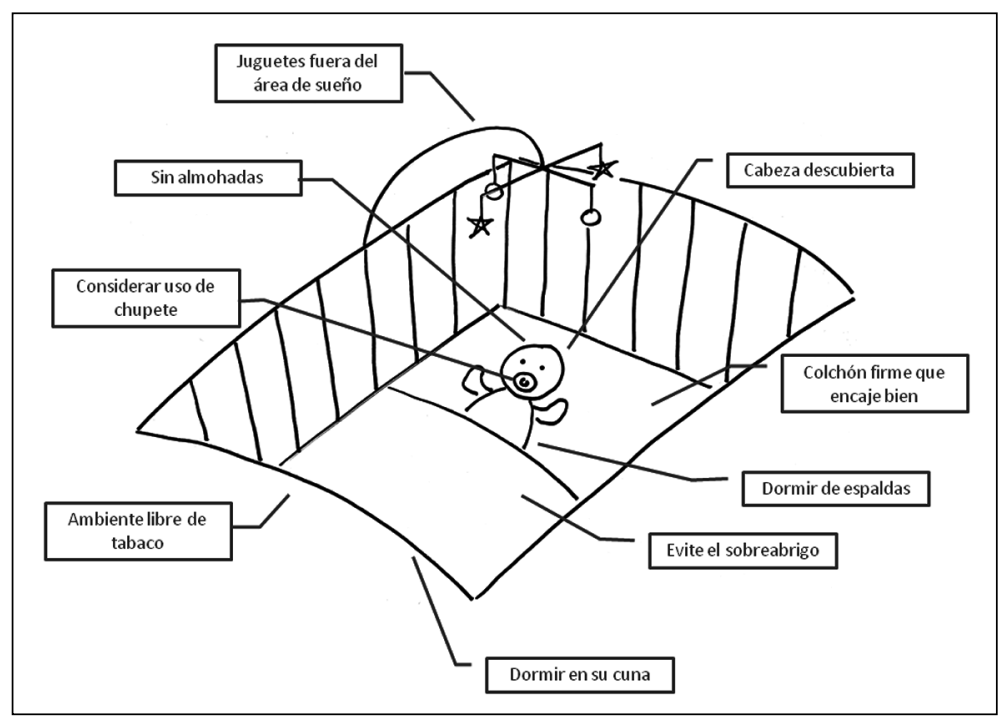

Figura 1. Tarjeta de Cuna.

Tabla 2. Principales Diferencias entre ALTE y SMSL

\begin{tabular}{|lll|}
\hline & SMSL & ALTE \\
\hline Incidencia x 1.000 RNV & $0,4-0,8$ & $0,6-2,5$ \\
Edad & $2-4$ meses & $2-3$ semanas antes \\
Género predominante & Masculino & Indiferente \\
Relación con sueño & Sueño & Vigilia o sueño \\
Posición de mayor riesgo & Prono & Indiferente \\
Efecto de campaña "Dormir de espaldas" & Reducción altamente significativa & Indiferente \\
\hline
\end{tabular}

La mayor parte de los estudios han determinado diferencias entre ellos (tabla 2); sin embargo, también se han demostrado similitudes, especialmente con respecto al tabaquismo materno que se encuentra significativamente aumentado en ambos grupos de lactantes y en los cuales también se han encontrado alteraciones polisomnográficas sutiles ${ }^{19-21}$.

\section{Estudio}

El estudio de laboratorio se plantea en casos en que el episodio se considera que fue amenazante de la vida, y en los que la historia y/o el examen físico no proporcionan una explicación del episodio que motivó la consulta. Así, por ejemplo, en un recién nacido con una coriza obstructiva, o en un lactante que presentó un laringoespasmo provocado por un episodio de reflujo gastroesofágico, como causas reconocibles del episodio que motivó la consulta, no sería necesario estudios de laboratorio (tabla 1-Flujograma) ${ }^{10,22-25}$.

\section{Evaluación inicial de laboratorio}

Incluye hemograma, gases en sangre, glicemia, electrolitos plasmáticos, nitrógeno ureico, calcio, magnesio, amonio, lactato, electroencefalograma, radiografía de tórax y electrocardiograma. En caso de haber compromiso sensorial, o algún déficit neurológico, debe realizarse un screening toxicológico (para tóxicos $\mathrm{y}$ fármacos $)^{22}$, y una imagen cerebral (ECO en menores de 3 meses, TAC o resonancia nuclear magnética).

\section{Evaluación secundaria de laboratorio}

Se considera sólo en casos seleccionados y debe ser guiada por una sospecha clínica que 


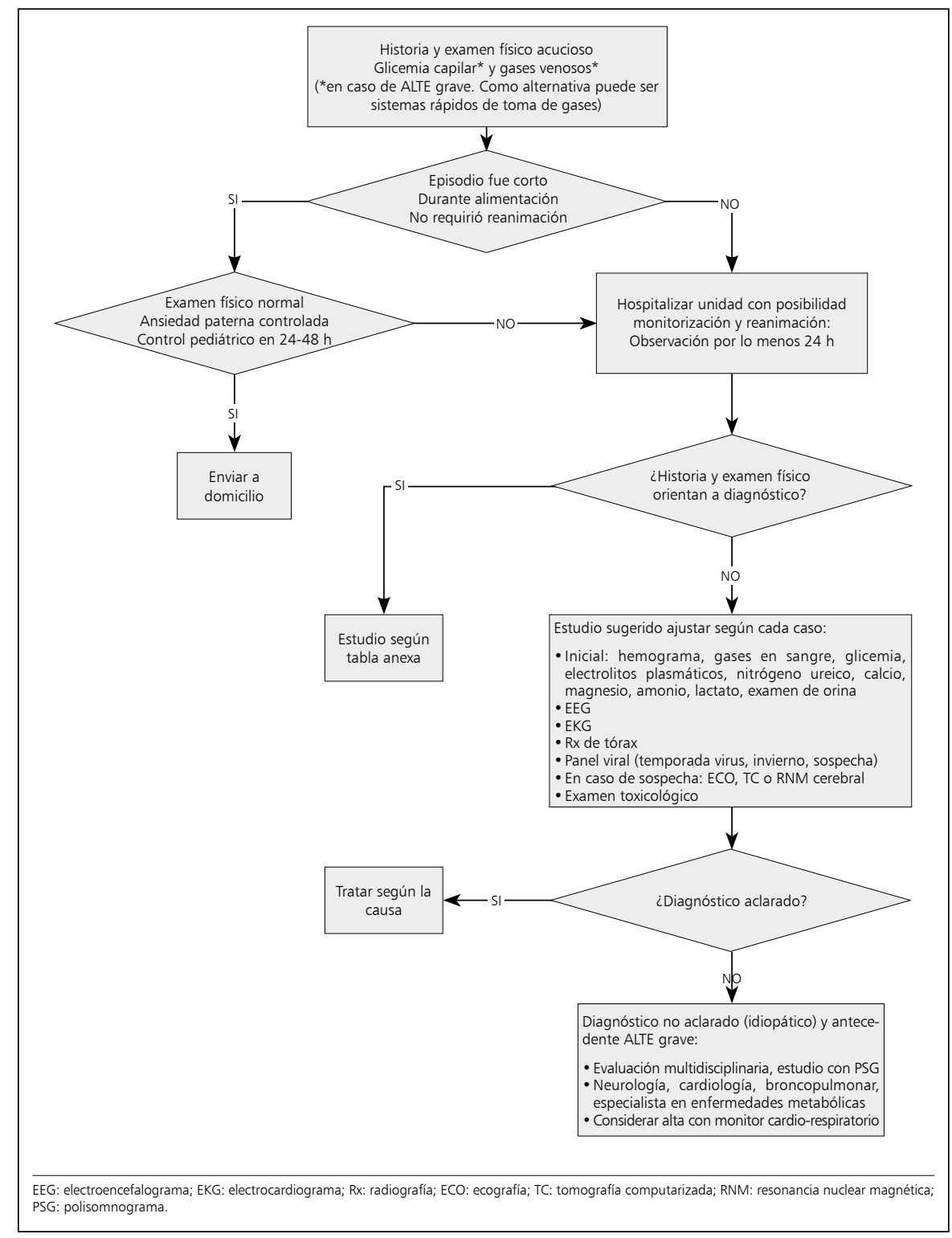

Flujograma de estudio de un paciente con ALTE.

oriente la investigación, pues la relación costobeneficio resulta extremadamente alta.

a. En paciente febril o de aspecto tóxico se debe realizar una evaluación completa según edad, hemocultivos, análisis completo de orina, estudio de LCR.

b. Si hay contexto de infección respiratoria aguda, investigación de virus respiratorios (IFD o amplificación genética), IFD o PCR Bordetella, radiografía de tórax.

c. En pacientes con atoros y ALTE recurrente, con sospecha de una alteración en la deglución, estudio de vídeo-deglución y una radiografía EEG con bario.

d. Sospecha de un anillo vascular: esófagograma con bario, fibrobroncoscopía, AngioTAC.

e. Frente a sospecha de enfermedad metabólica, por compromiso de conciencia, vómitos, deterioro neurológico, convulsiones, antecedentes familiares, debe realizarse un screening para enfermedades metabólicas y 


\begin{tabular}{|c|c|c|}
\hline & $\begin{array}{l}\text { Bradicardia } \\
\text { (Latidos/min) }\end{array}$ & $\begin{array}{c}\text { Apnea } \\
\text { (Duración segundos) }\end{array}$ \\
\hline $\begin{array}{l}\text { Prematuro (semanas edad gestacional corregida) } \\
\quad<40 \\
\quad 40-44 \\
>44\end{array}$ & $\begin{array}{r}100 \\
80 \\
70\end{array}$ & $\begin{array}{c}15 \\
15 \\
15-20\end{array}$ \\
\hline $\begin{array}{l}\text { Término (meses) } \\
\quad<1 \\
1-3 \\
3-12\end{array}$ & $\begin{array}{l}80 \\
70 \\
60\end{array}$ & $\begin{array}{l}20 \\
20 \\
20\end{array}$ \\
\hline
\end{tabular}

Figura 2. Recomendaciones de variables de monitor cardiorrespiratorio en niños prematuros y de término.

si los elementos clínicos y resultados preliminares lo ameritan, estudios específicos de enfermedades metabólicas.

f. Si hay sospecha de enfermedad cardíaca, electrocardiograma, Ecocardiografía. Si la sospecha se focaliza en una arritmia, se indica un Holter de arritmias de $24 \mathrm{~h}$.

g. Si hay elementos de trauma accidental o no accidental, convulsiones, procesos expansivos intracraneanos o hemorragias intracraneanas, está indicado realizar ECO (en menores de 3 meses), TAC o Resonancia nuclear magnética de cerebro.

h. Si hay sospecha de abuso infantil, además de una imagen cerebral se debe realizar estudio completo de esqueleto, fondo de ojo con dilatación pupilar y una evaluación del grupo familiar en sus aspectos sociales, psicológicos y factores de riesgo asociados.

i. En ALTEs que se presentan durante el sueño, asociados a una apnea, o con sospecha de una disfunción respiratoria, cardíaca o neurológica durante el sueño puede considerarse una polisomnografía, aunque este examen no ha demostrado ser útil en predecir nuevos episodios de ALTE, ni en reducir el riesgo de muerte súbita. También tiene indicación en pacientes portadores de malformaciones o síndromes genéticos, enfermedades neuromusculares o malformaciones cráneo-faciales.

\section{Uso de monitor y seguimiento}

\section{Seguimiento post alta}

Se sugiere que todos los pacientes deberían ser evaluados precozmente 48 a $72 \mathrm{~h}$ posterior al alta. Recomendamos en ALTE idiopático y/o de riesgo al menos 1 vez al mes durante mínimo 3 meses, luego según evolución de cada caso.

\section{Indicación de monitor cardiorrespiratorio}

Los monitores cardiorrespiratorios han sido usados en las últimas décadas para monitorizar pacientes con potencial riesgo de apneas o alteraciones de la frecuencia cardíaca. Su uso se expandió rápidamente después de la publicación de Steinschneider de 1972, en la cual se demostraba una asociación entre registros poligráficos con apneas previo al fallecimiento consecutivo de lactantes de una misma familia por SMSL ${ }^{13}$. Sin embargo, como se señaló anteriormente, se logró establecer con posterioridad que se trató de un infanticidio múltiple en este lamentable caso.

De todas formas, se debe tener presente algunas consideraciones respecto al monitor cardiorrespiratorio, antes de revisar sus indicaciones. El monitor cardiorespiratorio no detecta apneas obstructivas, ni episodios de caídas de saturación de $\mathrm{O}_{2}$ : detecta solamente apneas de tipo central y cambios en la frecuencia cardíaca (taquicardia, bradicardia). La monitorización cardiorrespiratoria no debe ser recomendada para prevenir la muerte súbita, ya que existe evidencia sólida que demuestra que su uso no la previene ${ }^{26}$.

En el estudio CHIME (Collaborative Home Infant Monitoring Evaluation $)^{26}$ que reclutó a más de 1.000 pacientes en California, se pudo demostrar similares tasas de muerte súbita en los grupos con y sin monitor cardiorrespiratorio. Por otro lado, está bien documentado que 
han existido casos de fallecimiento de pacientes debidamente conectados a un monitor cardiorrespiratorio ${ }^{27}$. Se propone además que el monitor cardiorrespiratorio o multiparámetros debe cumplir con las siguientes características (figura 2):

1. Registrar movimientos respiratorios y trazado electrocardiográfico.

2. Memoria para facilitar la lectura y validación de los eventos.

Los monitores que no sean cardiorrespiratorios y midan de otra forma movimientos del lactante sobre la cama o bien sean sobrepuestos no están recomendados formalmente.

Indicaciones sugeridas de monitor cardiorrespiratorio en pacientes que presentaron un ALTE:

1. ALTE idiopático, es decir, el cual en que no se ha logrado llegar a una etiología evidente, pese a un estudio acucioso y polisomnografía.

2. ALTE recurrente ( $\geq 2$ episodios).

3. ALTE grave, sin causa clara, con reanimación formal o cianosis persistente. Antecedentes de enfermedad de base (prematurez, displasia broncopulmonar, síndrome de Down, enfermedades neuromusculares, malformaciones cráneo-faciales, etc).

4. Pacientes con ALTE que requieran de ventilación u oxígeno $\left(\mathrm{O}_{2}\right)$ domiciliario.

5. Pacientes con ALTE y antecedente de ser hermano de un fallecido por muerte súbita.

La duración de la monitorización se propone por el siguiente tiempo:

1. Mínimo 6 semanas sin alarmas reales ni eventos, ausencia de apneas o bradicardias. No debe haber registros (revisados y validados) en la memoria del monitor que sean sugerentes de apneas, ni bradicardias reales, no artefactuales. La edad mínima para suspender el monitor son 3 meses de vida. En pacientes con factores de riesgo son 6 meses. Idealmente se debe realizar una polisomnografía previo a la suspensión de los monitores cardiorrespiratorios en aquellos pacientes con factores de riesgo, ALTE recurrente o idiopático.

2. En los pacientes que sean dependientes de
$\mathrm{O}_{2}$ o de ventilación asistida, deberá mantenerse el uso de monitor por todo el tiempo necesario mientras requieran de este tipo de soporte.

3. En aquellos pacientes que tengan el antecedente de un hermano fallecido por muerte súbita, al menos se deberá monitorizar hasta la edad en la cual el hermano(a) hubiera fallecido.

4. La utilidad del monitor pasado el 1 año de vida es desconocida y probablemente no aporte a la vigilancia de estos eventos. Sólo se sugiere seguir con monitor en casos muy puntuales, debidamente documentados con un estudio multidisciplinario y requirentes de soporte ventilatorio o de $\mathrm{O}_{2}$.

La lectura del monitor y seguimiento de los pacientes con ALTE, debe ser realizada de forma mensual inicialmente. Si se estimase necesario se sugiere evaluar eventos reportados por los padres de forma simultánea con el monitor.

Además se debe brindar un adecuado apoyo a los padres, con atención y ayuda psicológica. El uso de monitor cardiorrespiratorio se ha asociado a angustia y estrés en reportes como el estudio CHIME ${ }^{28}$, lo cual debe ser debidamente derivado y apoyado por salud mental. En casos que exista inseguridad respecto a la suspensión del monitor por parte de los padres, esto puede ser alargado dentro de un rango razonable. Se sugiere explicar las limitaciones del registro con un monitor cardiorrespiratorio para evitar generar una falsa seguridad o expectativas no reales respecto a su uso.

En todos los casos, se debe hacer una consejería sobre el dormir seguro y una capacitación a los padres y/o cuidadores en reanimación cardiopulmonar básica, ya que estas dos medidas han demostrado disminuir la muerte súbita. El lactante siempre debe dormir en su propia cuna en posición supina (de espaldas), en una superficie dura, no blanda (figura 1). Se debe evitar el uso de frazadas, el sobreabrigo, almohadas y cualquier elemento potencialmente riesgoso. Además, el médico tratante debe insistir en la necesidad de evitar el humo del tabaco y el colecho (dormir en la misma cama con los padres). 


\section{Referencias}

1.- Khushi A, Côté A: Apparent life-threatening events: assessment, risks, reality. Paediatr Respir Rev 2011; 12 (2): 124-132.

2.- National Institutes of Health Consensus Development: Conference on Infantile Apnea and Home Monitoring, Sept 29 to Oct 1, 1986. Pediatrics 1987; 79 (2): 292-9.

3.- Ross-Russell R, Ravikumar K: Apparent life-threatening Episodes in Children. Paediatrics and Child Health 2007; 17 (5): 188-92.

4.- McGovern MC, Smith MB: Causes of apparent life threatening events in infants: a systematic review. Archives of disease in childhood 2004; 89 (11): 1043-8.

5.- Esani N, Hodhman JE, Ehsani N, Hoppenbrouwers T: Apparent Life-Threatening Events and Sudden Infant Death Syndrome: Comparison of Risk Factors. J Pediatr 2008; 152 (3): 365-70.

6.- Kaji AH, Claudius I, Santillanes G, et al: Apparent LifeThreatening Event: Multicenter Prospective Cohort Study to Develop a Clinical Decision Rule for Admission to the Hospital. Ann Emerg Med 2013; 61 (4): 379-87.

7.- Fu LY, Moon RY: Apparent life-threatening events: an update. Pediatr Rev 2012; 33 (8): 361-8.

8.- Brockmann P, González X, Bertrand P, Sánchez I, Holmgren NI: Perfil clínico de lactantes hospitalizados por un episodio de ALTE (Apparent Life Threatening Event). Rev Chil Pediatr 2006; 77 (3): 267-73.

9.- Zenteno D, Quiroz G, Celis M, Tapia J: Causas atribuidas a eventos de aparente amenaza a la vida del lactate. Rev Chil Pediatr 2008; 79 (2): 163-71.

10.- Tieder JS, Altman RL, Bonkowsky JL, et al: Management of apparent life-threatening events in infants: a systematic review. J Pediatr 2013; 163 (1): 94-9.

11.- Al-Kindy HA, Gélinas JF, Hatzakis G, Côté A: Risk factors for extreme events in infants hospitalized for apparent life-threatening events. J Pediatr 2009; 154 (3) 332-7.

12.- Rocca $M$, et al: Episodio de posible amenaza a la vida- ALTE. Guía de Práctica Clínica 2005. Comité en Síndrome de muerte súbita del lactante Asociación Latino-Americana de Pediatría.

13.- Steinschneider A: Prolonged apnea and the sudden infant death syndrome: clinical and laboratory observations. Pediatrics 1972; 50 (4): 646-54.

14.- Tieder JS, Cowan CA, Garrison MM, Christakis DA: Variation in inpatient resourse utilization and management of apparent life-threatening events. J Pediatr 2008; 152 (5): 629-35.

15.- Santiago-Burruchaga M, Sánchez-Etxaniz J, Benito-
Fernández J, et al: Assessment and management of infants with apparent life-threatening events in the paediatric emergency department. Eur J Emerg Med 2008; 15 (4): 203-8

16.- Samuels M, Poets C, Noyes J, Hartmann H, Hervenston $J$, Southall D: Diagnosis and management after ALTE in infants and youg children who received cardiopulmonary resuscitation. BMJ 1993; 306: 489-92.

17.- Zenteno D: Eventos de aparente amenaza a la vida (ALTE): conceptos, etiología y manejo. Neumología Pediátrica 2006; 1 (3): 133-8.

18.- Kahn A: Recommended clinical evaluation of infants with an apparent life-threatening event. Consensus Document of the European Society for the Study and Prevention of Infant Death. Eur J Pediatr 2004; 163 : 108-15.

19.- Kiechl-Kohlendorfer U, Hof D, Peglow UP, TrawegerRavanelli B, Kiechl S: Epidemiology of apparent life threatening events. Arch Dis Child 2005; 90 (3): 297 300 .

20.- Edner A, Wennborg M, Alm B, Lagercrantz H: Why do ALTE infants not die in SIDS? Acta Paediatr 2007; 96 (2): 191-4.

21.- Franco P, Montemitro E, Scaillet $S$, et al: Fewer spontaneous arousals in infants with apparent life-threatening event. Sleep 2011; 34 (6): 733-43.

22.- Pitetti RD, Whitman E, Zaylor A: Accidental and nonaccidental poisonings as a cause of apparent life-threatening events in infants. Pediatrics 2008; 122: e359-62.

23.- Davies F, Gupta R: Apparent life threatening events in infants presenting to an emergency department. Emerg Med J 2002; 19 (1): 11-6.

24.- Brand DA, Altman RL, Purtill K, Edwards KS: Yield of diagnostic testing in infants who have had an apparent life-threatening event. Pediatrics 2005; 115 (4): 885-93.

25.- De Piero AD, Teach SJ, Chamberlain JM: ED evaluation of infants after an apparent life-threatening event. Am J Emerg Med 2004; 22 (2): 83-6.

26.- Hunt CE, Corwin MJ, Lister G, et al: Precursors of cardiorespiratory events in infants detected by home memory monitor. Pediatr Pulmonol 2008; 43 (1): 87-98.

27.- Poets A, Urschitz MS, Steinfeldt R, Poets CF: Risk factors for early sudden deaths and severe apparent life-threatening events. Archives of disease in childhood Fetal and neonatal edition 2012; 97 (6): f395-7.

28.- Silvestri JM, Lister G, Corwin MJ, et al: Factors that influence use of a home cardiorespiratory monitor for infants: the collaborative home infant monitoring evaluation. Archives of pediatrics \& adolescent medicine. 2005; 159 (1): 18-24. 